\title{
EPhIMM: computational workflow for fast phylogenetic inference based on multiple alignment of prokaryotic single-copy marker genes
}

\author{
Aleksei Korzhenkov \\ NRC Kurchatov Institute, Moscow, Russia \\ oscypek@ya.ru
}

\begin{abstract}
EPhIMM - a computational workflow for fast and accurate phylogenetic inference for prokaryotic genomes was developed and tested on bacterial and archaeal genomic datasets. Source code is freely available at https://github.com/laxeye/EPhIMM.
\end{abstract}

Keywords - genomics, phylogenetics, workflow

Motivation and aim

Motivation

Multiple sequence alignment (MSA) and phylogenetic inference are one of the most time- and resource-consuming operations in bioinformatical analysis. The growing number of complete and draft prokaryotic genomes enforces search of an effective computational workflow for phylogenetic analysis

Aim

The aim of the study was to select and test a combination of bioinformatic tools for sequence search, alignment and phylogenetic inference, each of them could be used on a common PC or laptop and create a workflow for user-friendly phylogenetic analysis.

\section{Methods}

41 protein coding prokaryotic single copy marker genes that have HMM profiles were used from CheckM software [1]. CDS were predicted using prodigal [2] with default settings whether genomic sequences were used. CDS for marker genes were found using hmmsearch [3] with default setting except e-value $=1 \mathrm{e}-10$ against corresponding HMM profiles from pfam [4]. Sequences were extracted using eslsfetch tool from easel software package (https:/github.com/EddyRivasLab/easel). When several protein coding sequences from one genome matched same HMM profile a sequence with lowest mean distance to all other sequences was selected using BioKotlin (https://github.com/laxeye/BioKotlin).

Three MSA tools were tested with default settings: Clustal Omega [5], Muscle [6] and MAFFT [7]. Columns, containing more than $50 \%$ of gaps were removed from the resulting alignments using BioKotlin.

Three tools for phylogenetic tree inference were tested: RAxML [8], PhyML [9] and FastTree claimed to be several magnitudes faster than RAxML and PhyML [10]. LG substitution matrix was used for all tools. CAT approximation with 25 and 20 rate categories for RaxML and FastTree respectively was used. Phylogenetic trees were visualized and topologies of the trees were compared using ete3 [11].

Bash script was written to manage transition of user data from one tool to another.
Three sets of high quality complete genomes (one genome per species) of type strains were analyzed: Bacillaceae, Euryarchaeota and Gammaproteobacteria. Genomes of Staphylococcus aureus subsp. aureus DSM 20231, Sulfolobus acidocaldarius DSM 639 and Campylobacter fetus subsp. fetus NCTC 10842 were used as outgroups respectively. Totally 39, 117 and 332 genomes were in the first, second and third set respectively. Predicted protein coding sequences were downloaded from NCBI RefSeq Release 95.

\section{Results}

A PC with 8 GB DDR3 and Intel Core i7-4790 3.60GHz with Ubuntu 18.04 operating system was used to test the worflow. RAM usage appeared to be the main restriction: PhyMl required more than $8 \mathrm{~GB}$ on Gammaproteobacteria dataset, while Clustal Omega was unable to perform MSA on all datasets, for data on time consumption see Table 1 .

Phylogenetic analysis of 117 genomes on reference PC took roughly 10 minutes resulting in phylogenetic tree with mean support value of nodes boo.

Result of the work is EPhiMM - a workflow including tools for protein prediction (prodigal), marker gene search and extraction (hmmer and easel), multiple sequence alignment (MAFFT) and phylogenetic tree inference (FastTree) coupled with helper tool (BioKotlin). EPhIMM may use nucleotide genomic sequences or sets of predicted proteins. The workflow depends on mentioned tools and Java virtual machine 8 which could be easily installed using Bioconda. Source code and documentation are freely available at https:/github.com/laxeye/EPhIMM under MIT license.

Table 1. Phylogenetic inference based on aminoacid sequences

\begin{tabular}{|c|c|c|c|}
\hline Genomic set & Bacillaceae & Euryarchaeota & $\begin{array}{c}\text { Gammapro- } \\
\text { teobacteria }\end{array}$ \\
\hline $\begin{array}{c}\text { Genome } \\
\text { count }\end{array}$ & 39 & 117 & 332 \\
\hline $\begin{array}{c}\text { Extraction of } \\
\text { marker } \\
\text { genes, m:s }\end{array}$ & $0: 31.2$ & $1: 27.9$ & $4: 56.5$ \\
\hline $\begin{array}{c}\text { Sequence } \\
\text { alignment } \\
\text { (MAFFT } \\
\text { FFT-NS-2), } \\
\text { m:s }\end{array}$ & $0: 53.8$ & $5: 55.1$ & $15: 47.2$ \\
\hline $\begin{array}{c}\text { Sequence } \\
\text { alignment } \\
\text { (MUSCLE), } \\
\text { m:s }\end{array}$ & $4: 51.4$ & $20: 05.9$ & $70: 49.3$ \\
\hline
\end{tabular}




\begin{tabular}{|c|c|c|c|}
\hline $\begin{array}{c}\text { Alignment } \\
\text { length, aa }\end{array}$ & 30452 & 39007 & 63616 \\
\hline $\begin{array}{c}\text { Proportion } \\
\text { of gaps in } \\
\text { alignment, \% }\end{array}$ & 49.32 & 66.56 & 73.36 \\
\hline $\begin{array}{c}\text { Phylogenetic } \\
\text { tree } \\
\text { inference } \\
\text { (FastTree), } \\
\text { m:s }\end{array}$ & $0: 53.1$ & $2: 58.6$ & $10: 07.6$ \\
\hline $\begin{array}{c}\text { Phylogenetic } \\
\text { tree } \\
\text { inference } \\
\text { (RAxML), } \\
\text { h:m:s }\end{array}$ & $0: 26: 12$ & $3: 8: 16$ & $19: 21: 38$ \\
\hline $\begin{array}{c}\text { Phylogenetic } \\
\text { tree } \\
\text { inference } \\
\text { (PhyML), } \\
\text { m:s }\end{array}$ & $14: 51.68$ & $123: 28.4$ & N/A \\
\hline
\end{tabular}

\section{ACKNOWLEDGMENT}

Supported by a grant of Ministry of Science and Higher Education of Russian Federation allocated to the Kurchatov Center for Genome Research (075-15-2019-1659).

\section{REFERENCES}

[1] Parks, D. H. et al. (2015). CheckM: assessing the quality of microbial genomes recovered from isolates, single cells, and metagenomes. Genome research, 25(7), 1043-1055.

[2] Hyatt, D. et al. (2010). Prodigal: prokaryotic gene recognition and translation initiation site identification. BMC bioinformatics, 11(1), 119.

[3] Eddy, S. R. (2011). Accelerated profile HMM searches. PLoS computational biology, 7(10), e1002195.

[4] Finn, R. D. et al. (2015). The Pfam protein families database: towards a more sustainable future. Nucleic acids research, 44(D1), D279-D285.

[5] Sievers, F. et al. (2011). Fast, scalable generation of high-quality protein multiple sequence alignments using Clustal Omega. Molecular systems biology, 7(1).

[6] Edgar, R. C. (2004). MUSCLE: multiple sequence alignment with high accuracy and high throughput. Nucleic acids research, 32(5), 17921797.

[7] Katoh, K., Standley, D. M. (2013). MAFFT multiple sequence alignment software version 7: improvements in performance and usability. Molecular biology and evolution, 30(4), 772-780.

[8] Stamatakis, A. (2014). RAxML version 8: a tool for phylogenetic analysis and post-analysis of large phylogenies. Bioinformatics, 30(9), 1312-1313.

[9] Guindon, S. et al. (2010). New algorithms and methods to estimate maximum-likelihood phylogenies: assessing the performance of PhyML 3.0. Systematic biology, 59(3), 307-321.

[10] Price, M.N., Dehal, P.S., and Arkin, A.P. (2010) FastTree 2 Approximately Maximum-Likelihood Trees for Large Alignments. PLOS ONE, 5(3):e9490.

[11] Huerta-Cepas, J., Serra, F., Bork, P. (2016). ETE 3: reconstruction, analysis, and visualization of phylogenomic data. Molecular biology and evolution, 33(6), 1635-1638. 